\title{
Morbilidad en Recién Nacidos de Término en Relación a su Edad Gestacional
}

\author{
MARCELO ARMADANS ${ }^{2}$, MARIA FABIANA OSSORIO ${ }^{1}$, CARLOS PEDICONE $^{2}$, \\ PABLO DURÁN $^{1}$, FERNANDO FERRERO ${ }^{1}$ \\ 1. Hospital de Niños Pedro de Elizalde, Buenos Aires, Argentina. \\ 2. Instituto Argentino de Diagnóstico y Tratamiento, Buenos Aires, Argentina.
}

\begin{abstract}
Morbidity Risk and Gestational Age Among Full-Term Newborns

Introduction: Newborns of Gestational Age (GA) $\geq 37$ weeks are considered to be full-term, but they might show increased morbidity. Objective: To evaluate morbidity risk between newborns 37-38 weeks GA vs those over 39 weeks. Patients and Methods: Cohort study of all children born at a private clinic in Buenos Aires between January 1, 2006 and July 31, 2007, product of simple pregnancies and without major congenital abnormalities. A total of 1829 children met the criteria, among which 823 (45\%) were 37-38 weeks GA, and $1006(55 \%)$ were 39 weeks or over. The following parameters were recorded: birth route, maternal history and morbidity. Morbidity included at least one of the following: respiratory distress $>2$ hours post-birth, use of intravenous solutions, jaundice and use of antibiotics. The association between morbidity and GA was evaluated using Chi-square, and logistical regression was used to evaluate the relationship between newborn morbidity and GA, birth route or maternal hypertension. Results: Newborns of 37-38 weeks GA showed higher incidence of each component of morbidity: respiratory distress $(\mathrm{OR}=2,55 \mathrm{IC} 95 \%=1,70-3,82)$, jaundice $(\mathrm{OR}=2,24$ IC95\% 1,72-2,29), antibiotic use (OR = 2,31 IC 95\% = 1,15-4,69) and IV use (OR=2,29 IC95\%=1,57-3,33). Multivariate analysis showed that GA 37-38 weeks $(\mathrm{OR}=1,89 \mathrm{IC} 95 \%=1,31-2,71)$ and a C-section $(\mathrm{OR}=$ $1,65$ IC95\% $=1,18-2,32)$ constituted independent predictors of morbidity. Conclusion: In this experience, a gestacinal age under 39 weeks increases morbidity risk.
\end{abstract}

(Key words: Gestational age, mortality, risk, newborn, full-term).

Rev Chil Pediatr 2010; 81 (5): 402-408

\section{RESUMEN}

Introducción: A pesar de considerarse recién nacidos (RN) de término a aquellos con edad gestacional (EG) $\geq 37$ semanas, aún dentro de este grupo, los de menor EG podrían presentar mayor morbilidad. Objetivo: Evaluar si existen diferencias en la frecuencia de morbilidad entre recién nacidos de 37-38 semanas de EG y aquellos de EG $\geq$ de 39 semanas. Pacientes y Método: Estudio de cohorte incluyendo todos los RN de una institución privada de Buenos Aires, nacidos entre 01/01/06 y 31/07/07, con EG $\geq 37$ semanas, producto de

Trabajo recibido el 21 de abril de 2010, devuelto para corregir el 06 de julio de 2010, segunda versión el 28 de julio de 2010 , aceptado para publicación el 16 de agosto de 2010.

Correspondencia a:

Maria Fabiana Ossorio

E-mail:mossorio@intramed.net 
gestas simples y sin malformaciones congénitas mayores $(n=1829)$. De ellos, $823(45 \%)$ tenían EG 37-38 semanas y $1006(55 \%)$ EG $\geq 39$ semanas. Se registró vía de nacimiento, antecedentes maternos y morbilidad (presencia de al menos una de las siguientes condiciones: dificultad respiratoria $>2$ horas del nacimiento, aporte de líquidos intravenosos, ictericia y uso de antibióticos). Se evaluó asociación entre morbilidad (en general y para cada uno de sus componentes) y EG por medio de Chi cuadrado y se utilizó regresión logística para evaluar asociación entre EG, vía de parto (cesárea o vaginal), hipertensión arterial materna y morbilidad. Resultados: Los RN de 37-38 semanas presentaron mayor prevalencia de cada componente de morbilidad: dificultad respiratoria $(\mathrm{OR}=2,55 \mathrm{IC} 95 \%=1,70-3,82)$, ictericia $(\mathrm{OR}=2,24 \mathrm{IC} 95 \% 1,72-2,29)$, uso de antibióticos $(\mathrm{OR}=2,31$ IC 95\%=1,15-4,69) y accesos vasculares $(\mathrm{OR}=2,29 \mathrm{IC} 95 \%=1,57-3,33)$. El análisis multivariado mostró que EG 37-38 semanas $(\mathrm{OR}=1,89 \mathrm{IC} 95 \%=1,31-2,71)$ y el parto por cesárea $(\mathrm{OR}=1,65$ IC95\% $=1,18-2,32)$ constituían predictores independientes de morbilidad. Conclusión: En recién nacidos de término, la EG < 39 semanas incrementa la morbilidad.

(Palabras clave: Edad gestacional, mortalidad, riesgo, recién nacido, término).

Rev Chil Pediatr 2010; 81 (5): 402-408

\section{Introducción}

El manejo perinatal de los embarazos de riesgo ha cambiado en los últimos años, obteniéndose mejores resultados en la evolución de los prematuros de menor edad gestacional (EG). Al mismo tiempo, el parto prematuro se ha incrementado notoriamente, en su mayor parte a expensas de nacimientos producidos entre 34 y 36 semanas de gestación ${ }^{1}$.

Estos neonatos presentan mayor morbimortalidad con el consiguiente incremento en los costos de salud. Eventos tales como dificultad respiratoria, inestabilidad térmica, hipoglucemia y problemas alimentarios, son más frecuentes en este rango de edad gestacional ${ }^{2}$.

Por otra parte, los recién nacidos de 37 y 38 semanas, aunque considerados de término, también pueden presentar mayor riesgo de complicaciones cuando se comparan con aquellos de edad gestacional mayor. La práctica obstétrica y neonatal considera a este último grupo de pacientes como funcionalmente de término y su manejo es encarado en consecuencia. Sin embargo, esta actitud podría no siempre ser apropiada, ya que la experiencia clínica encuentra a estos niños cada vez con mayor frecuencia en las unidades de neonatología ${ }^{3}$.

Nuestro objetivo fue evaluar si existían diferencias en la frecuencia de morbilidad entre los recién nacidos de 37-38 semanas de edad gestacional y aquellos de edad gestacional $\geq$ de 39 semanas.

\section{Pacientes y Métodos}

Se diseñó un estudio de cohorte incluyendo todos los recién nacidos con edad gestacional igual o mayor a 37 semanas e inferior a 43 semanas, nacidos entre 01 de enero de 2006 y 31 de julio de 2007 en una institución privada de la ciudad de Buenos Aires $(n=1829)$. En dicha institución se efectúan aproximadamente 1400 partos anuales y su servicio de terapia intensiva neonatal cuenta con 10 plazas. Fueron excluidos aquellos recién nacidos producto de una gesta múltiple, que presentaran malformaciones congénitas mayores o edad gestacional incierta (según fecha de última menstruación).

En todos los recién nacidos incluidos en el estudio se registró peso al nacer (en gramos), edad gestacional estimada por fecha de última menstruación, vía de nacimiento (parto vaginal o cesárea), presencia de hipertensión o diabetes materna y morbilidad del recién nacido definida por la presencia de al menos una de las siguientes condiciones:

- Dificultad respiratoria que persistiera luego de 2 horas desde el nacimiento, manifestada por quejido, aleteo nasal, taquipnea, retracción costal o requerimiento de oxígeno suplementario por cualquier método.

- Requerimiento de aporte de líquidos endovenosos.

- Administración de antibióticos en cualquier momento de la internación, por cualquier motivo y duración. 
- Ictericia que hubiera requerido fototerapia (según guías de la Academia Americana de Pediatría ${ }^{4}$.

Toda la información requerida para el estudio fue obtenida de los registros de la institución.

Se comparó días de internación y peso de nacimiento entre grupos de EG ( $\geq 39$ semanas y 37-38 semanas) por medio de prueba de $\mathrm{T}$ o Mann-Whitney, según correspondiera (se evaluó ajuste a normalidad por la prueba de Kolmogorov-Smirnov). Se evaluó la asociación entre morbilidad (en general, y para cada uno de sus componentes) y grupos de EG por medio de la prueba de $\chi^{2}$. Además, se incluyeron grupos de EG, morbilidad, vía de nacimiento, hipertensión materna y bajo peso para la edad gestacional en un modelo de regresión logística, junto con un término de interacción entre grupos de EG y vía de nacimiento. Se estimaron los Odds Ratio (OR) y sus correspondientes intervalos de confianza (IC 95\%). Se asumió un nivel de significación de $\mathrm{p}<0,05$. Los cálculos se efectuaron con SPSS 11.5.1 (SPSS Inc, Chicago, 2002) y StatCalc 6.0, 1993.

De acuerdo a lo contemplado en la Ley 25326 de la República Argentina (Ley de Protección de Datos Personales), dado que la información fue obtenida de los registros de la institución y se preservó la identidad de los titulares de los datos mediante un mecanismo de disociación adecuado (sólo se identificaban por número de registro), no se requirió consentimiento informado de los representantes legales. Se aseguró la confidencialidad de los datos y el proyecto se llevó a cabo respetando las normas vigentes en investigación clínica, sin potenciales conflictos de interés que declarar.

\section{Resultados}

En la institución se registraron 1932 nacimientos de $\mathrm{RN} \geq 37$ semanas de EG durante el período de estudio. Se excluyeron 103 casos (67 gestas múltiples, 31 FUM incierta y 5 malformaciones congénitas), por lo que la población quedó constituida por 1829 recién nacidos, de ellos 823 (45\%) tenían EG 37-38 semanas y 1006 (55\%), EG $\geq 39$ semanas.
El 3,9\% de la población fue categorizada como bajo peso para la edad gestacional. En relación a patología en las madres, 3,7\% presentaban antecedentes de hipertensión y 1,1\% de diabetes.

El peso de nacimiento fue mayor en el grupo con $E G \geq 39$ semanas que en el de EG 37-38 semanas ( $3462 \pm 402 \mathrm{~g}$ vs $3177 \pm 400$ g; $\mathrm{p}<0,0001$ ), mientras que la mediana de la duración de la internación fue 3 días en ambos grupos $(p=0,046)$.

La vía de parto fue cesárea en 948 nacimientos $(51,8 \%)$. Al analizar la misma según edad gestacional, encontramos que fue cesárea en $493(59,9 \%)$ de los RN de 37-38 semanas y en $455(45,2 \%)$ de los RN de más de 39 semanas de EG $(\mathrm{p}<0,001$; OR $=1,8 \mathrm{IC} 95 \%$ $=1,49-2,19)$. La cesárea fue más frecuente en aquellos casos con antecedentes de hipertensión $(\mathrm{p}>0,001 ; \mathrm{OR}=6,0 \mathrm{IC} 95 \%=2,8-13,08)$, pero no en los de diabetes $(\mathrm{p}=0,11 ; \mathrm{OR}=2,43$ IC95\% $=0,71-9,08$ ).

La prevalencia de morbilidad en toda la población fue 21,38\%. El 6,67\% de la población presentó dificultad respiratoria, $15,85 \%$ presentó ictericia, $2,18 \%$ requirió uso de antibióticos y 7,27 \% uso de fluidos intravenosos. No se registraron decesos.

La prevalencia de morbilidad en general según edad gestacional fue $29,3 \%$ en el grupo de $37-38$ semanas y $14,9 \%$ en el grupo de RN $\geq 39$ semanas de EG $(\mathrm{p}<0,0001 ; \mathrm{OR}=2,36$ IC95\% $=1,87-2,99$ ). Al comparar la morbilidad en general y cada una de las morbilidades específicas entre ambos grupos en el análisis bivariado se encontró que todas presentaban una frecuencia significativamente mayor en los RN de 37-38 semanas (tabla 1).

Al llevar a cabo el análisis multivariado a través de regresión logística con un modelo que incluyó EG (según grupo), vía de nacimiento, hipertensión materna, bajo peso para la edad gestacional y un término de interacción entre grupo de EG y vía de nacimiento, se observó que EG, vía de nacimiento y bajo peso para la edad gestacional fueron predictores independientes de morbilidad. La EG 37-38 semanas mostró un OR $=1,89(\mathrm{IC} 95 \%=1,31-2,71, \mathrm{p}$ $<0,001)$, el nacimiento por cesárea $\mathrm{OR}=1,65$ (IC95\% $=1,18-2,32 ; \mathrm{p}<0,003)$, el bajo peso 
MORBILIDAD EN RECIÉN NACIDOS DE TÉRMINO

Tabla 1. Distribución de los hallazgos según grupo de edad gestacional

\begin{tabular}{|lrrrrrrr|} 
& \multicolumn{2}{c}{$\mathbf{3 7 - 3 8}$} & & $\mathbf{3 9}$ & OR & IC 95 \% & p \\
& n & \% & n & \% & & & \\
Cesárea & 493 & 59,9 & 455 & 45,2 & 1,81 & $1,49-2,19$ & $<0,0001$ \\
Morbilidad & 241 & 29,3 & 150 & 14,9 & 2,36 & $1,87-2,99$ & $<0,0001$ \\
\hline Dificultad respiratoria & 81 & 9,8 & 41 & 4,1 & 2,57 & $1,72-3,86$ & $<0,0001$ \\
Ictericia & 179 & 21,7 & 111 & 11,0 & 2,24 & $1,72-2,92$ & $<0,0001$ \\
Antibióticos & 26 & 3,2 & 14 & 1,4 & 2,31 & $1,15-4,69$ & 0,01 \\
Líquidos endovenosos & 88 & 10,7 & 50 & 5,0 & 2,29 & $1,57-3,33$ & $<0,001$ \\
\hline
\end{tabular}

para la edad gestacional OR $=2,19(\mathrm{IC} 95 \%=$ $1,33-2,71 ; \mathrm{p}=0,002)$ y el término de interacción entre $\mathrm{EG}$ y vía de nacimiento $\mathrm{OR}=0,99$ $(\mathrm{IC} 95 \%=0,62-1,58 ; \mathrm{p}=0,99)$ (prueba de Hosmer-Lemeshow $=0,98$ ).

\section{Discusión}

Nuestro estudio evidenció que, dentro de los recién nacidos considerados de término, aquellos de 37-38 semanas de edad gestacional presentaron mayor morbilidad que aquellos con edad gestacional igual o mayor a 39 semanas.

La edad gestacional al nacimiento ha disminuido en las últimas décadas. Los partos prematuros en EE.UU. se incrementaron de 9,1\% en 1981 a $12,3 \%$ en el año $2003^{1}$. El aumento puede deberse en algunos casos a la decisión de algunos obstetras de interrumpir el embarazo para evitar mayor riesgo en determinadas patologías en las que antes se prefería mantener el embarazo ${ }^{5}$. Es muy posible que esto también se vea influenciado por la elevada sobrevida que hoy presentan los prematuros, sobre todo a partir de las 33 semanas. Sin embargo, existen algunos motivos de interrupción del embarazo que podrían evitarse y de esta forma disminuir el impacto que estos nacimientos producen en los sujetos y en la salud pública.

La disminución en la edad gestacional al nacimiento ha llevado a la aparición del fenómeno conocido como prematuro tardío o "late preterm". Esto incluye RN de 35-36 semanas de EG los cuales, pese a hallarse cercanos al término, presentan mayor morbimortalidad que los de término. El estudio de esta condi- ción ha merecido la atención de varias investigaciones $^{1,2,6,7}$.

Menos estudiado ha sido el fenómeno del RN de término temprano o "early term". Esta categoría incluye RN de 37-38 semanas de EG y es probable que compartan al menos parte del espectro de complicaciones de los "late preterm". Dado que el número de nacimientos en este grupo es mucho mayor, el impacto de este fenómeno en la salud pública es potencialmente, también, mucho mayor ${ }^{3,8}$.

A pesar de las diferencias que pueden existir, nuestros resultados pueden ser, de alguna forma, homologables a lo observado en los "prematuros tardíos". En este sentido, Wang y colaboradores encontraron importantes diferencias en los resultados clínicos al comparar recién nacidos casi a término ("prematuros tardíos") con aquellos de mayor edad gestacional, ya que los neonatos de menor edad gestacional presentaron más problemas médicos documentados a pesar de presentar peso adecuado y buena vitalidad estimada por el puntaje de Apgar 6 .

En nuestro estudio, el grupo de RN de 3738 semanas de EG presentó morbilidad respiratoria significativamente superior comparada con los $\mathrm{RN} \geq 39$ semanas (OR: 2,57). Esto coincide con lo referido por Zhang quien observó que los RN de 37 semanas de EG presentaban el doble de riesgo de requerir ventilación mayor de 30 minutos que los RN de 40 semanas de $\mathrm{EG}^{3}$. Por otro lado, Wax refiere que los niños nacidos de 37-38 semanas de gestación evidencian un riesgo significativamente mayor de requerir ventilación mecánica (37 semanas: $\mathrm{OR}=38,5$ y 38 semanas: $\mathrm{OR}=13,3)^{9}$; 
sin embargo, la amplitud de los intervalos de confianza del $95 \%(8,3-178,3$ y $2,8-64,0$, respectivamente), muestran probablemente un tamaño muestral restringido para dar precisión a dichos resultados.

Desde los estudios de Usher ${ }^{10,11}$ es conocido el impacto del nacimiento por cesárea en la morbilidad neonatal. Parilla describe mayor incidencia de dificultad respiratoria en niños nacidos por cesárea, especialmente a edad gestacional menor ${ }^{12}$ y Levine describe una incidencia casi 5 veces mayor de hipertensión pulmonar en neonatos nacidos por cesárea que en partos vaginales ${ }^{13}$. Recientemente, en un importante estudio de cohorte, Hansen demostró que los $\mathrm{RN}$ de término nacidos por cesárea electiva presentan mayor riesgo de morbilidad respiratoria que los nacidos por vía vaginal o cesárea de emergencia y que dicho riesgo se incrementa con la disminución en la edad gestacional $^{14}$. El mismo fenómeno fue verificado en nuestro medio por Ceriani Cernadas, al encontrar que los niños de término nacidos por cesárea presentaban más riesgo de presentar morbilidad respiratoria que aquellos nacidos por vía vaginal ${ }^{15}$. A pesar de la evidencia antes mencionada, en nuestro estudio encontramos que el riesgo de morbilidad asociado a cesárea en niños de término fue independiente de la edad gestacional.

Al igual que con la patología respiratoria, también la hiperbilirrubinemia podría mostrar mayor incidencia en $\mathrm{RN}$ cercanos al término ${ }^{1}$. Maisels y colaboradores encontraron que la edad gestacional entre 35 y 36 tanto como entre 37 y 38 semanas, constituyen factores de riesgo para reinternación por ictericia en el período neonatal cuando se comparaban con edad gestacional mayor ${ }^{16}$. En nuestro estudio, los RN de 37-38 semanas presentaron el doble de riesgo de requerir luminoterapia que los de $\geq 39$ semanas.

Aunque la restricción del crecimiento intrauterino se mostró como predictor independiente de morbilidad en el recién nacido, su baja prevalencia $(3,9 \%)$ en relación a la elevada prevalencia de recién nacido de "término temprano" (45\%), hace que posiblemente tenga una limitada trascendencia en el fenómeno estudiado.
A pesar que los RN con EG 37-38 semanas presentaron una duración de la internación igual a aquellos con $E G \geq 39$ semanas, no debe olvidarse que los costos de una internación que incluye administración de fluidos, antibióticos, oxígeno o luminoterapia son sensiblemente mayores. Dado que el grupo de RN con EG 37-38 presentó el doble de morbilidad y que su prevalencia fue muy elevada ( $45 \%$ ) es razonable pensar que este fenómeno puede ocasionar un impacto trascendente en los costos de salud.

Nuestro estudio posee potenciales limitaciones que deben ser tenidas en cuenta. Por un lado, el mismo se desarrolló en una institución privada donde el público asistido presenta una muy elevada proporción de adecuado control del embarazo y pertenece a un nivel socioeconómico superior al que se asiste habitualmente en las instituciones públicas, por lo cual se debe verificar si lo observado se cumple en otros escenarios.

Con respecto al análisis de la patología materna, sólo incluyó hipertensión arterial y diabetes, sin embargo estas son las entidades que más frecuentemente provocan complicaciones en el embarazo. Por otra parte, a pesar que la patología materna (hipertensión) se asoció a mayor tasa de cesáreas, esta condición no se mostró como predictor independiente de morbilidad en el recién nacido. No se consideró si en los casos de nacimiento por cesárea ésta había sido electiva o con trabajo de parto previo. Existe evidencia que podría indicar que el trabajo de parto previo podría disminuir la posibilidad de morbilidad en el recién nacido ${ }^{17}$.

Finalmente, la tasa de cesáreas observada en nuestro estudio merece una consideración especial. La misma ha crecido alarmantemente en los últimos 30 años, superando en algunos países al $40 \%$ de los nacimientos ${ }^{18}$. Esto ha preocupado a la OMS, quien ha propuesto como objetivo mantenerla por debajo del $15 \%{ }^{19}$. El estudio de este fenómeno es complicado y escapa al objetivo del presente trabajo. Sin embargo, pueden mencionarse algunos factores que influirían en esta realidad, incluyendo mejoramiento de las técnicas quirúrgicas y anestésicas, percepción de médicos y pacientes sobre la seguridad del procedimiento, temor a juicios por responsabilidad profesio- 
nal y, en algunos casos, elección de la mujer ("cesárea a demanda"). Tampoco puede soslayarse el impacto que el uso generalizado del monitoreo fetal intraparto podría haber tenido en su incremento. En Argentina, no existe una estimación nacional sobre la tasa de cesáreas, pero un estudio en el sector público la sitúa en $22,8 \%{ }^{20}$. Sin embargo, nuestro estudio se desarrolló en un escenario completamente diferente (sector privado, clase media-alta), donde, según los mismos autores, sería esperable una tasa de cesáreas considerablemente mayor.

A pesar de las consideraciones mencionadas, dada su magnitud y la posibilidad de ser prevenido, el fenómeno del RN de término temprano merece atención por su potencial impacto en la salud pública, además de las consecuencias directas en la salud de una importante proporción de los recién nacidos.

\section{Conclusiones}

Los recién nacidos de 37 y 38 semanas de edad gestacional presentaron mayor morbilidad que los nacidos a partir de las 39 semanas. La ictericia, uso de líquidos endovenosos, uso de antibióticos y dificultad respiratoria fueron significativamente mayores en este grupo cuando se los comparó con los nacidos en la semana 39 o más.

En recién nacidos de término, menor edad gestacional, bajo peso al nacer y nacimiento por cesárea fueron predictores independientes de morbilidad. Estudios más amplios son necesarios para verificar estos datos y, de comprobarse, efectuar las recomendaciones tendientes a controlar la situación.

\section{Agradecimientos}

Al Dr. Santiago Orozco, por garantizar el acceso a los datos.

\section{Referencias}

1.- Raju T, Higgins R, Stark A, Leveno K: Optimizing Care and Outcome for Late-Preterm (Near-Term) Infants: A Summary of the Workshop Sponsored by the National
Institute of Child Health and Human Developement. Pediatrics 2006; 118: 1207-14.

2.- Yoder B, Gordon M, Barth W: Late preterm birth: does the changing obstetric paradigm alter the epidemiology of respiratory complications? Obstet Ginecol 2008; 111 (4): 814-22.

3.- Zhang $X$, Kramer M: Variations in mortality and morbidity by gestational age among infants born at term. J Pediatr 2009; 154 (3): 358-62.

4.- American Academy of Pediatrics Subcommittee on Hyperbilirubinemia: Management of Hyperbilirubinemia in the Newborn Infant 35 or More Weeks of Gestation Pediatrics 2004; 114: 297-316.

5.- Caughey AB, Musci TJ: Complications of term pregnancies beyond 37 weeks of gestation. Obstet Gynecol 2004; 103 (1): 57-62.

6.- Wang ML, Dorer DJ, Catlin EA: Clinical outcomes of near-term infants. Pediatrics 2004; 114 (2): 372-6.

7.- Barrington KJ, Finer NN: Recent advances: care of near term infants with respiratory failure. BMJ 1997; 315: 1215-8.

8.- Reddy U, Ko C, Willinger M: "Early" term births (37-38 weeks) are associated with increased mortality. Am J Obst Gynecol 2006; 195 (6): S202

9.- Wax JR, Herson V, Carignan E, Mather J, Ingardia CJ: Contribution of elective delivery to severe respiratory distress at term. Am J Perinatol 2002; 19: 81-6.

10.- Usher R, McLean F, Maughan GB: Respiratory distress syndrome in infants delivered by cesarean section. Am J Obstet Gynecol 1964; 88: 806-15.

11.- Usher R, Allen AC, McLean FH: Risk of respiratory distress syndrome related to gestational age, route of delivery, and maternal diabetes. Am J Obstet Gynecol 1971; 111: 826-32.

12.- Parrilla BV, Dooley $S L$, Jansen RD, Socol ML: Iatrogenic respiratory distress syndrome following elective repeat cesarean delivery. Obstet Gynecol 1993; 81: 392-5.

13.- Levine EM, Ghai V, Barton JJ, Strom CM: Mode of delivery and risk of respiratory diseases in newborns. Obstet Gynecol 2001; 97: 439-42.

14.- Hansen AK, Wisborg $K$, Uldbjerg $N$, Henriksen TB: Risk of respiratory morbidity in term infants delivered by elective caesarean section: cohort study. BMJ 2008; 336 (7635): 85-7.

15.- Ceriani Cernadas JM, Mariani G, Pardo A, et al: Nacimiento por cesárea al término en embarazos de bajo riesgo: efectos sobre la morbilidad neonatal. Arch Argent Pediatr 2010; 108 (1): 17-23.

16.- Maisels $M$, Kring E: Length of stay, jaundice, and hos- 
ARMADANS M. y cols.

pital readmission. Pediatrics 1998; 101: 995-8.

17.- Engle $W$, Kominiarek $M$ : Late Preterm Infants, Early Term Infants, and Timing of Elective Deliveries. Clin Perinatol 2008; 35: 325-41.

18.- Betrán A, Merialdi M, Lauer J, et al: Rates of caesarean section: analysis of global, regional and nacional Estimates. Paediatric and Perinatal Epidemiology 2007; 21
(98): 113.

19.- World Health Organization: Appropriate technology for birth. Lancet 1985; 326 (8452): 436-7.

20.- Casale R: Niveles socioeconómicos y tasa de cesárea en la Argentina. ¿Una inequidad en el acceso a la salud? Estudio ecológico. Rev Obstet Ginecol B Aires 2009; 88 (982): 200-11. 